Europhys. Lett., 29 (9), pp. 693-698 (1995)

\title{
On the Validity of a Variational Principle for Far-from-Equilibrium Steady States.
}

\author{
A. Santos $(*)$, V. Garzó $(*)$ and J. J. BREY $(* *)$ \\ $\left(^{*}\right)$ Departamento de Física, Universidad de Extremadura - E-06071 Badajoz, Spain \\ (**) Física Teórica, Universidad de Sevilla - E-41080 Sevilla, Spain
}

(received 18 October 1994; accepted in final form 23 February 1995)

PACS. $47.50+d-$ Non-Newtonian fluid flows.

PACS. 05.20.Dd - Kinetic theory.

PACS. $05.60+\mathrm{w}-$ Transport processes: theory.

\begin{abstract}
It is shown that an exact solution of the non-linear Boltzmann equation does not verify the variational principle for non-equilibrium steady states proposed by Evans and Baranyai (Phys. Rev. Lett., 67 (1991) 2597). Therefore, the principle does not provide a basis for the understanding of far-from-equilibrium steady states.
\end{abstract}

A few years ago, Evans and Baranyai (EB) [1] proposed a variational principle to characterize non-equilibrium steady states. The EB principle is formulated for systems arbitrarily far from equilibrium and reduces to the principle of minimum-entropy production [2] for near-equilibrium situations. To support their hypothesis, Evans and Baranyai provided simulation data for a thermostatted dense fluid under uniform shear flow. Within statistical uncertainties, the data agreed with the predictions of the principle.

Given the essential role that a variational principle would play in the development of a general theory for far-from-equilibrium systems, it is very important to investigate the validity of the EB principle. In a previous paper [3], we used the BGK model kinetic equation [4] to analyse it, and found significant, although small, deviations. Nevertheless, since the BGK equation is a simplified model of the Boltzmann equation, no definitive conclusion about the validity of the principle was obtained. The aim of this letter is to carry out a similar analysis, but now using the non-linear Boltzmann equation, and without introducing any kind of approximation. In this way, the calculations we present here can be considered as exact in the context of kinetic theory.

As in ref. [1] and [3], we consider a fluid under uniform shear flow. This state is macroscopically characterized by a constant density, $n$, a spatially homogeneous temperature, $T$, and a linear profile of the $x$-component of the velocity field along the $y$-direction, i.e. $u_{i}(\boldsymbol{r})=a_{i j} r_{j}, a_{i j}=a \delta_{i x} \delta_{j y}$, where $a$ is the constant shear rate. Besides, in order to compensate for the viscous heating effects and obtain a steady state, a drag force of the form $-\alpha \boldsymbol{V}$ is introduced [5]. Here $\boldsymbol{V}=\boldsymbol{v}-\boldsymbol{u}$ is the peculiar velocity and the thermostat parameter $\alpha$ is determined from the condition that the temperature remains constant.

In short, the EB principle states [1] that the average of the phase-space compression

(C) Les Editions de Physique 
factor, $A$, which is a measure of the rate of change of the volume of space phase [5], is a local maximum with respect to variations of endogenous variables. Following again ref. [1] and [3], we will consider the fourth velocity moment

$$
K_{4}=\int \mathrm{d} \boldsymbol{V}\left(V_{x}^{4}+V_{y}^{4}+V_{z}^{4}\right) f(\boldsymbol{r}, \boldsymbol{v}, t)
$$

as the endogenous variable, $f(\boldsymbol{r}, \boldsymbol{v}, t)$ being the one-particle distribution function. The idea now is to compute $A$ for systems having different prescribed values of $K_{4}$ and check whether it has a maximum in the absence of constraints on $K_{4}$. To constrain the value of $K_{4}$, another nonconservative force, this time proportional to the cube of the components of the peculiar velocity, is introduced. Therefore, each particle in the system is subjected to an external force $\boldsymbol{F}$ with components

$$
F_{i}=-\alpha V_{i}-\beta V_{i}^{3}, \quad i=x, y, z
$$

As said above, the parameters $\alpha$ and $\beta$ are adjusted to keep both the temperature and $K_{4}$ fixed at prescribed values. For our discussion it will be convenient to take $a$ and $\beta$ as independent variables, instead of $a$ and $K_{4}$. Aside from a constant factor, the phase-space compression factor for our system reads [5]

$$
\Lambda(\alpha, \beta)=-\left(\alpha+\frac{3 k_{\mathrm{B}} T}{m} \beta\right)
$$

The EB principle implies that

$$
\left.\lambda(a) \equiv\left(\frac{\partial \Lambda}{\partial \beta}\right)_{a}\right|_{\beta=0}=0
$$

for arbitrary shear rates.

Our goal is to exactly evaluate $\lambda(a)$ for a low-density gas. We start from the Boltzmann equation describing the thermostatted uniform shear flow state with a constrained value of $K_{4}[6]$ :

$$
-a V_{y} \frac{\partial}{\partial V_{x}} f-\frac{1}{m} \frac{\partial}{\partial V_{i}}\left(\alpha V_{i}+\beta V_{i}^{3}\right) f=J[f, f],
$$

where $J[f, f]$ is the Boltzmann collision operator. From this equation it follows that the velocity moments

$$
M_{k_{1}, k_{2}, k_{3}} \equiv \frac{1}{n} \int \mathrm{d} \boldsymbol{V} V_{x}^{k_{1}} V_{y}^{k_{2}} V_{z}^{k_{3}} f(\boldsymbol{V})
$$


obey the hierarchy

$$
\begin{aligned}
& a k_{1} M_{k_{1}-1, k_{2}+1, k_{3}}+\frac{\alpha}{m}\left(k_{1}+k_{2}+k_{3}\right) M_{k_{1}, k_{2}, k_{3}}+ \\
& \quad+\frac{\beta}{m}\left(k_{1} M_{k_{1}+2, k_{2}, k_{3}}+k_{2} M_{k_{1}, k_{2}+2, k_{3}}+k_{3} M_{k_{1}, k_{2}, k_{3}+2}\right)=J_{k_{1}, k_{2}, k_{3}},
\end{aligned}
$$

where

$$
J_{k_{1}, k_{2}, k_{3}}=\frac{1}{n} \int \mathrm{d} \boldsymbol{V} V_{x}^{k_{1}} V_{y}^{k_{2}} V_{z}^{k_{3}} J[f, f]
$$

Now we expand the several quantities in powers of $\beta$ by writing

$$
\begin{aligned}
\alpha(a, \beta) & =\alpha_{0}(a)+\alpha_{1}(a) \beta+\ldots, \\
M_{k_{1}, k_{2}, k_{3}}(a, \beta) & =M_{k_{1}, k_{2}, k_{3}}^{(0)}(a)+M_{k_{1}, k_{2}, k_{3}}^{(1)}(a) \beta+\ldots, \\
J_{k_{1}, k_{2}, k_{3}}(a, \beta) & =J_{k_{1}, k_{2}, k_{3}}^{(0)}(a)+J_{k_{1}, k_{2}, k_{3}}^{(1)}(a) \beta+\ldots
\end{aligned}
$$

The coefficients in these expansions are nonlinear functions of the shear rate. Inserting these expansions into eq. (7) one gets a set of hierarchies, one for each order in $\beta$. Those corresponding to the two lowest orders are

$$
a k_{1} M_{k_{1}-1, k_{2}+1, k_{3}}^{(0)}+\frac{\alpha_{0}}{m}\left(k_{1}+k_{2}+k_{3}\right) M_{k_{1}, k_{2}, k_{3}}^{(0)}=J_{k_{1}, k_{2}, k_{3}}^{(0)},
$$

$$
\begin{aligned}
a k_{1} M_{k_{1}-1, k_{2}+1, k_{3}}^{(1)}+ & \frac{\alpha_{0}}{m}\left(k_{1}+k_{2}+k_{3}\right) M_{k_{1}, k_{2}, k_{3}}^{(1)}+\frac{\alpha_{1}}{m}\left(k_{1}+k_{2}+k_{3}\right) M_{k_{1}, k_{2}, k_{3}}^{(0)}+ \\
& +\frac{1}{m}\left[k_{1} M_{k_{1}+2, k_{2}, k_{3}}^{(0)}+k_{2} M_{k_{1}, k_{2}+2, k_{3}}^{(0)}+k_{3} M_{k_{1}, k_{2}, k_{3}+2}^{(0)}\right]=J_{k_{1}, k_{2}, k_{3}}^{(1)} .
\end{aligned}
$$

Equation (10) is the moment hierarchy for the unconstrained state. Once its solution is known, eq. (11) gives the moments to first order in $\beta$. Then, the coefficients $\alpha_{0}$ and $\alpha_{1}$, which are the quantities needed to get the first two terms of the $\beta$-expansion of $\Lambda$, are determined from the constant-temperature condition, i.e. $M_{2,0,0}+M_{0,2,0}+M_{0,0,2}=3 k_{\mathrm{B}} T / m$. Therefore, the moments we are interested in are those appearing in the above expression. Thus far the description is general, valid for any interaction potential. In order to get explicit results, we will restrict ourselves to Maxwell molecules (i.e. particles interacting via an $r^{-4}$ potential). In that case, $J_{k_{1}, k_{2}, k_{3}}$ can be expressed in terms of moments of degree equal to or less than $k_{1}+k_{2}+k_{3}$. In particular,

$$
J_{2,0,0}=-v\left(M_{2,0,0}-\frac{p}{m n}\right)
$$

and

$$
J_{1,1,0}=-v M_{1,1,0}
$$


where $p=n k_{\mathrm{B}} T$ is the pressure and $\nu$ is an effective collision frequency. In the special case of Maxwell molecules, the hierarchies (10) and (11) can be exactly solved in a recursive way. In order to evaluate $\lambda(a)$, it is sufficient to obtain the second-degree moments $\left(k_{1}+k_{2}+k_{3}=2\right)$ from eq. (11). In this way, one easily gets

$$
\begin{aligned}
& M_{0,0,2}^{(1)}=-\frac{1}{1+2 \alpha_{0}}\left(2 \alpha_{1} M_{0,0,2}^{(0)}+2 M_{0,0,4}^{(0)}\right), \\
& M_{0,2,0}^{(1)}=-\frac{1}{1+2 \alpha_{0}}\left(2 \alpha_{1} M_{0,2,0}^{(0)}+2 M_{0,4,0}^{(0)}\right), \\
& M_{1,1,0}^{(1)}=-\frac{1}{1+2 \alpha_{0}}\left(a M_{0,2,0}^{(1)}+2 \alpha_{1} M_{1,1,0}^{(0)}+M_{3,1,0}^{(0)}+M_{1,3,0}^{(0)}\right), \\
& M_{2,0,0}^{(1)}=-\frac{1}{1+2 \alpha_{0}}\left(2 a M_{1,1,0}^{(1)}+2 \alpha_{1} M_{2,0,0}^{(0)}+2 M_{4,0,0}^{(0)}\right) .
\end{aligned}
$$

Here and below we take units such that $v=1, m=1$, and $2 k_{\mathrm{B}} T / m=1$. From the consistency condition $M_{2,0,0}^{(1)}+M_{0,2,0}^{(1)}+M_{0,0,2}^{(1)}=0$, it is now a matter of straightforward calculations to get the expression of $\alpha_{1}$, and from it compute $\lambda=-\alpha_{1}-3 / 2$. The result is

$$
\begin{aligned}
\lambda=\frac{2}{3} \frac{1+2 \alpha_{0}}{1+6 \alpha_{0}}\left[M_{4,0,0}^{(0)}+\right. & M_{0,0,4}^{(0)}-\frac{3}{2}+\left(1+6 \alpha_{0}\right)\left(M_{0,4,0}^{(0)}-\frac{3}{4}\right)- \\
& \left.\quad-3 \alpha_{0}\left(1+2 \alpha_{0}\right)\left(\frac{M_{1,3,0}^{(0)}+M_{3,1,0}^{(0)}}{a}+\frac{3}{2}\right)+9 \alpha_{0}^{2} \frac{3+2 \alpha_{0}}{1+2 \alpha_{0}}\right],
\end{aligned}
$$

where use has been made of the expressions for the unconstrained second-degree moments. By substituting the explicit shear-rate dependence of the fourth-degree moments recently derived [7], it turns out that $\lambda$ is different from zero for all nonvanishing values of the shear rate $a$. In particular, in the limit of small $a$ it is easily found that

$$
\lambda(a) \approx \frac{3}{5} \frac{7 \nu^{\prime}-8}{\nu^{\prime}} \alpha_{0},
$$

where $v^{\prime} \simeq 1.873$ is an eigenvalue of the linearized Boltzmann equation associated to fourth-degree moments. Since for small shear rates $\alpha_{0} \approx a^{2} / 3$, it follows that $\lambda \approx 0.55 a^{2}$. Therefore, the EB principle is only verified to first order in the shear rate, i.e. in the Navier-Stokes approximation. In other words, it has the same range of validity as the well-known principle of minimum-entropy production [2]. It is worth mentioning that in the case of the BGK model equation $\lambda(a)$ vanishes up to third order in $a$ (super-Burnett approximation). More concretely, we found that $\lambda^{\mathrm{BGK}} \approx 31 a^{4} / 3$ [3]. This different behavior is due to the discrepancies in the fourth moments for the unconstrained system [8]. In fact, eq. (14) also holds for the BGK model equation.

In order to deeper analyse the foundations of the EB principle, we have also studied the $\beta$-dependence of the shear viscosity $\eta=-2 M_{1,1,0} / a$. There are at least two reasons which render this study interesting. First, the shear viscosity is the most relevant physical quantity characterizing the nonequilibrium behavior of the system. Secondly, since both $A$ and $\eta$ are proportional to the entropy production in the Navier-Stokes regime, the comparison of their behaviours provides a test of whether the average of the phase-space 


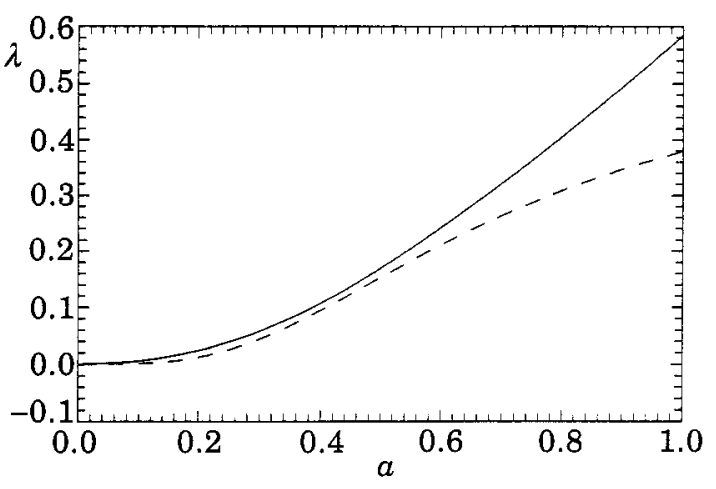

Fig. 1.

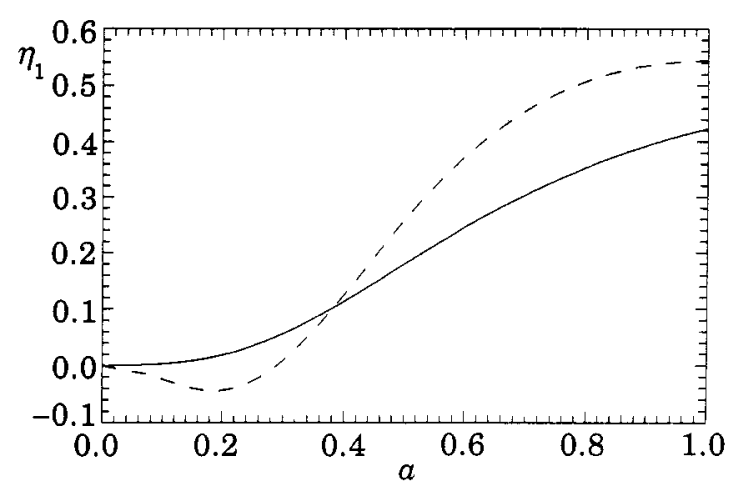

Fig. 2.

Fig. 1. - Shear rate dependence of $\lambda$ as obtained from the Boltzmann equation (solid line) and from the BGK equation (dashed line).

Fig. 2. - Shear rate dependence of $\eta_{1}$ as obtained from the Boltzmann equation (solid line) and from the $\mathrm{BGK}$ equation (dashed line).

compression factor really plays a special role upon formulating a variational principle to describe the steady state. The $\beta$ expansion of $\eta$ is

$$
\eta=\eta_{0}+\eta_{1} \beta+\ldots
$$

where

$$
\begin{gathered}
\eta_{0}=\left(1+2 \alpha_{0}\right)^{-2}, \\
r_{1}=\frac{2}{3 \alpha_{0}\left(1+2 \alpha_{0}\right)^{2}}\left[M_{4,0,0}^{(0)}+M_{0,4,0}^{(0)}+M_{0,0,4}^{(0)}-\frac{9}{4}-\frac{3}{2} \lambda\right] .
\end{gathered}
$$

In the small shear rate limit,

$$
\eta_{1} \approx 3 \frac{49197 v^{\prime 2}-79268 v^{\prime}-14700}{12005 v^{\prime 2}} \alpha_{0} \simeq 0.22 a^{2} .
$$

On the other hand, from the BGK equation one gets $\eta_{1}^{B G K} \approx-3 a^{2}[3]$. Therefore, while for the BGK equation a variational principle based on $\Lambda$ is better than one based on $\eta$, in the sense that the former leads to results that are exact up to a higher order in the shear rate, such a difference does not exist in the case of the Boltzmann equation. Even more, the discrepancies found from the Boltzmann equation to $a^{2}$ order are larger for $\Lambda$ than for $\eta$. Of course, these results are restricted to variations of the fourth moment $K_{4}$.

Figures 1 and 2 show the functions $\lambda(a)$ and $\eta_{1}(a)$, respectively. Both the Boltzmann and the BGK expressions have been plotted. From fig. 1 is again verified that, for finite values of the shear rate, the EB principle is more accurate to describe the solution of the BGK model equation than the solution of the Boltzmann equation. Nevertheless, the opposite happens when the shear viscosity is considered. It must also be noticed that the Boltzmann equation leads to an expression for $\eta_{1}$ that monotonically increases with $a$, while $\eta_{1}^{\mathrm{BGK}}$ presents a minimum around $a \simeq 0.2$.

In summary, we have analysed the EB variational principle for the phase-space compression factor from a solution of the non-linear Boltzmann equation for uniform shear 
flow. The results presented here are exact to all orders in the shear rate. We have proved that the EB principle is not obeyed by the Boltzmann equation beyond the Navier-Stokes order. Consequently, the principle cannot be considered as a solid basis to develop a general theory of far-from-equilibrium steady states. In particular, it is not clear why the phase-space compression factor $\Lambda$ should be a good candidate for the formulation of a variational principle.

Partial support from the Dirección General de Investigación Científica y Técnica (Spain) through Grants No. PB91-0316 (AS and VG) and No. PB92-0683 (JJB) is gratefully acknowledged.

\section{REFERENCES}

[1] Evans D. J. and Baranyai A., Phys. Rev. Lett., 67 (1991) 2597.

[2] DE Groot S. R. and Mazur P., Non-Equilibrium Thermodynamics (Dover, New York) 1984.

[3] Brey J. J., Santos A. and Garzó V., Phys. Rev. Lett., 70 (1993) 2730.

[4] FERZIGer J. and KAPER H., Mathematical Theory of Transport Processes in Gases (North-Holland, Amsterdam) 1972.

[5] Evans D. J. and Morriss G. P., Statistical Mechanics of Nonequilibrium Liquids (Academic, London) 1990.

[6] Dufty J. W., Santos A., Brey J. J. and Rodríguez R. F., Phys. Rev. A, 33 (1986) 459.

[7] Santos A. and Garzó V., Physica A, 213 (1995) 409.

[8] Garzó V. and Santos A., Physica A, 213 (1995) 426. 